\title{
Flight test performance of a high precision navigation Doppler Lidar
}

\author{
Diego Pierrottet $^{\mathrm{a}}$, Farzin Amzajerdian ${ }^{\mathrm{b}}$, Larry Petway ${ }^{\mathrm{b}}$, Bruce Barnes ${ }^{\mathrm{b}}$, George Lockard ${ }^{\mathrm{b}}$ \\ ${ }^{a}$ Coherent Applications, Inc., 20 Research Dr., Hampton VA, 23666 \\ ${ }^{\mathrm{b}}$ NASA Langley Research Center, Hampton VA 23681
}

\begin{abstract}
A navigation Doppler Lidar (DL) was developed at NASA Langley Research Center (LaRC) for high precision velocity measurements from a lunar or planetary landing vehicle in support of the Autonomous Landing and Hazard Avoidance Technology (ALHAT) project. A unique feature of this DL is that it has the capability to provide a precision velocity vector which can be easily separated into horizontal and vertical velocity components and high accuracy line of sight (LOS) range measurements. This dual mode of operation can provide useful information, such as vehicle orientation relative to the direction of travel, and vehicle attitude relative to the sensor footprint on the ground. System performance was evaluated in a series of helicopter flight tests over the California desert. This paper provides a description of the DL system and presents results obtained from these flight tests.
\end{abstract}

Key words: Doppler Lidar, vector velocity, navigation Lidar, Laser Remote Sensing

\section{INTRODUCTION}

ALHAT is developing a navigation Doppler Lidar (DL) to support precision pin point landing on the lunar surface. The DL is a versatile instrument capable of providing precision velocity vectors relative to the sensor reference frame, vehicle platform altitude, and ground relative attitude. With this sensor the landing vehicle can acquire a lunar surface inertial navigation fix during the approach phase, accurate to a few centimeters in position and a few centimeters per second in velocity. This allows the vehicle to accurately navigate from a few kilometers altitude to the previously defined surface location very accurately.

The breadboard navigation DL was the device under test for the ALHAT Field Test, where the DL was installed aboard a helicopter and tested over the California desert. This field test had a total of six flights; four flights over a flat dry lake bed and two over rough, hilly terrain. The data collected during FT2 proved to be valuable in demonstrating the capabilities of the DL, and also serves as a tool to test and develop signal processing and analysis algorithms. Analysis of the data shows velocity measurements in excellent agreement with the high accuracy GPS derived velocities. Ground relative altitude and attitude measurements were also demonstrated. This paper covers a sensor description, state vector computation methodology, and results obtained from helicopter flight tests.

\section{DL WAVEFORM DESCRIPTION}

Description of the DL concept and some preliminary ground measurements were reported previously ${ }^{1,2,3}$ and are briefly repeated here. The DL has two operational modes defined by the waveform of the transmitter - a continuous wave (CW) mode and a frequency modulated continuous wave (FMCW) mode. The desired mode of operation can be selected at any time by the user.

The lidar obtains high-resolution range and velocity information from a FMCW laser waveform whose instantaneous frequency is modulated linearly with time. Figure 1 shows the waveform's frequency content versus time, and the resulting intermediate frequency (IF) that holds the desired range and velocity information. The green triangular waveform represents the frequency content of the transmitted waveform, and the blue trace simulates a received 


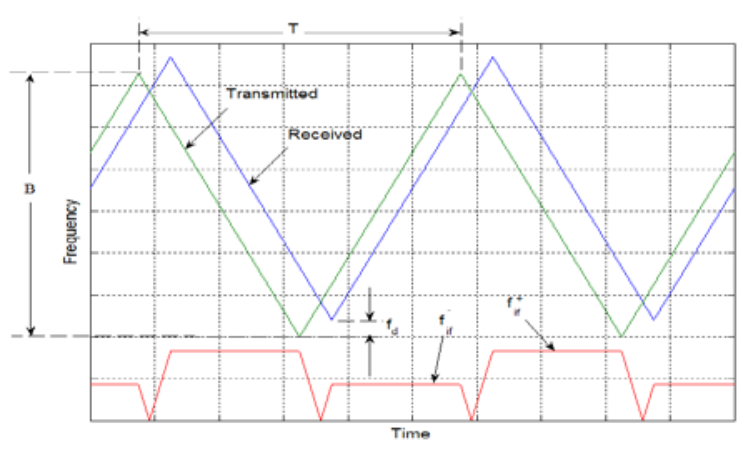

Figure 1 The sawtooth curves represent the frequency content of the DL waveform versus time. Received waveform is delayed in time. Lower trace is the difference between transmit and receive waveforms. waveform. The horizontal shift to the right of the received waveform is due to the time delay $\left(t_{r}\right)$ caused by the round trip time of flight of the laser beam to the target. The vertical shift of the received waveform represents the Doppler frequency change that arises from the motion of the vehicle relative to the target (ground).

The DL design uses an optical homodyne receiver configuration, in which a portion of the transmitted beam serves as the reference local oscillator (LO) for the optical receiver. The LO optical field mixes with the time delayed received field at the detector yielding a time varying intermediate frequency (IF) as shown by the lower (red) trace in Figure 1. The IF trace shows two distinct frequencies, one caused by the up-ramp ( $\mathrm{f}_{\mathrm{IF}}$ ), and one caused by the down-ramp $\left(\mathrm{f}^{+}{ }_{\mathrm{IF}}\right)$ of the waveform. The Doppler frequency in terms of the two Ifs is:

$$
f_{d}=\frac{f_{i f}^{+}-f_{i f}^{-}}{2}
$$

The IFs generated from the time delays (range) of the received waveforms are :

$$
f_{R}=\frac{f_{i f}^{+}+f_{i f}^{-}}{2}
$$

which is directly related to the target range by the equation:

$$
R=\frac{T C}{4 B} f_{R}
$$

where $\mathrm{R}$ is the range to target, $\mathrm{B}$ is the modulation bandwidth, $\mathrm{T}$ is the waveform period, and $\mathrm{c}$ is the speed of light.

\section{DL SYSTEM DESCRIPTION}

Figure 2 is a block diagram that illustrates the configuration of the all fiber DL. An FMCW waveform is generated from a very narrow line width seed laser. The seed laser modulated output is directed through a single mode fiber to a high

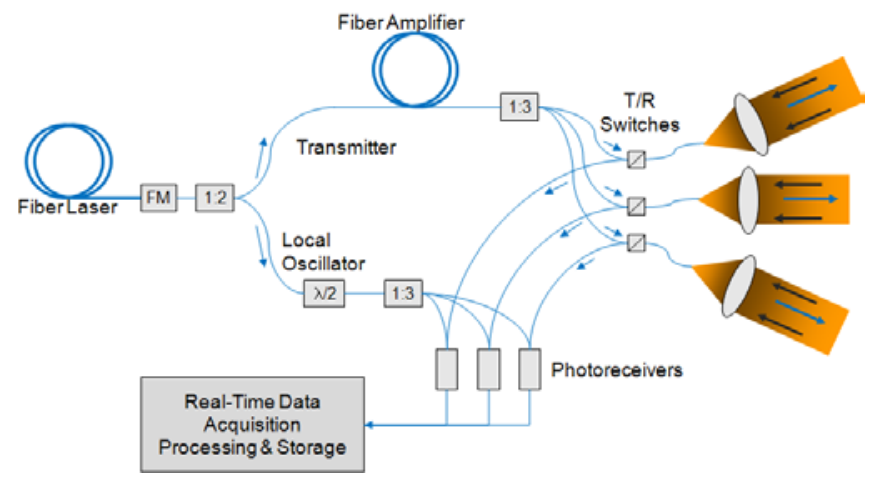

Figure 2 DL system block diagram. power fiber amplifier. Line width measurements at the output of the amplifier show negligible line broadening by the amplifier. The output of the fiber amplifier is split into three components in order to distribute the power to three optical channels mounted on the sensor's optical head. The optical head consists of three fiber-tofree space coupling telescopes, which were mounted separately from the rest of the system inside a gimbal on the nose of the helicopter. Signals from the ground are collected by these telescopes and sent to three pairs of heterodyne photoreceivers via optical fiber cables. The outputs of the detectors are processed by a Pentium Dual 


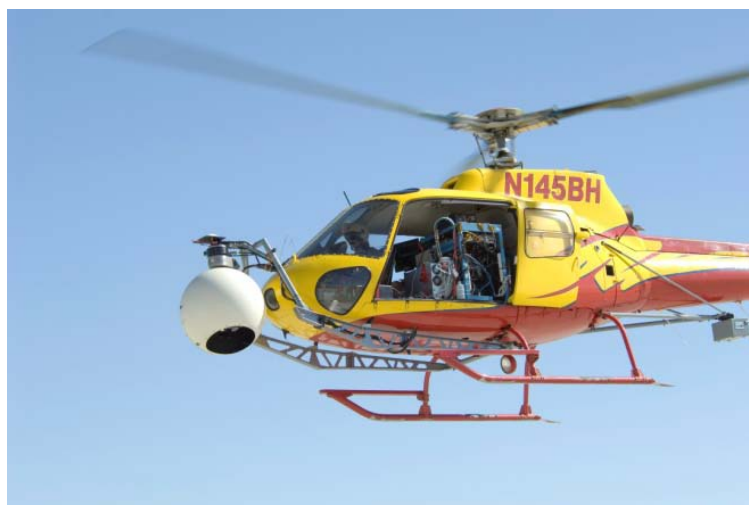

Figure 3 The DL optical head is mounted on the gimbal at the nose of the helicopter.
Core processor based receiver, capable of storing the temporal data for post processing, and providing real-time range and velocity measurements to be displayed on a graphical user interface (GUI). Real-time range and velocity measurement is a critical capability necessary for precision navigation. All system electronics and fiber optic components are housed in a standard instrumentation rack. Figure 3 is a photograph of the Eurocopter AS350D helicopter with the DL during one of the flights. The DL optical head is mounted inside the white gimbal spherical shroud, which is pointing in the nadir position while collecting data.

The telescopes inside the optical head are connected to the transmitter and receiver through a $25 \mathrm{ft}$ long fiber optic cable.

During data collection, the gimbal points downward towards nadir and the telescopes align as defined in the geometric description described next.
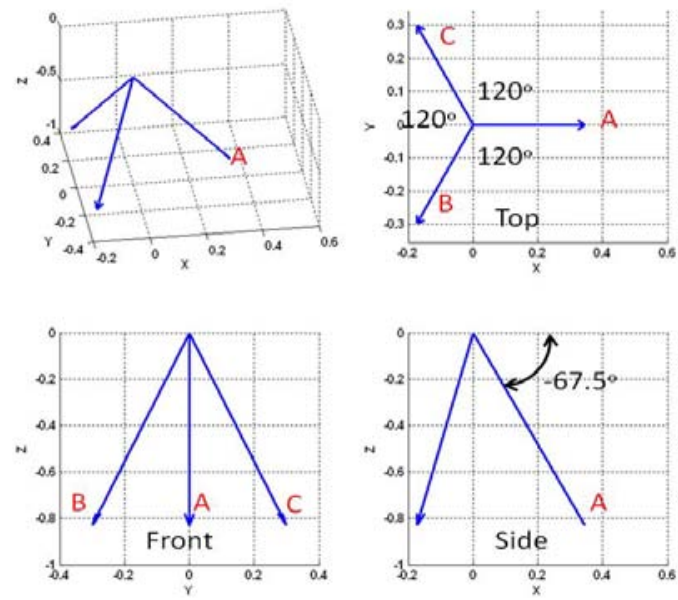

Figure 4 Unit vectors describing the sensor geometry. Helicopter forward direction is positive $\mathrm{x}$ - axis, up is positive $\mathrm{z}$ - axis. Unit vectors A, B, and C corresponds to each of the three sensor optical channels.

\subsection{Geometric description}

In the following geometric description, one can assume that the sensor frame of reference and the vehicle or platform frame of reference are the same. The geometry of the DL is shown in Figure 4. At the sensor reference frame, $\hat{x}, \hat{y}, \hat{z}$ are unit vectors in the $\mathrm{x}-, \mathrm{y}-$, and $\mathrm{z}$-axes respectively. The sensor has three transmit/receive optical channels that collect line of sight (LOS) range and velocity measurements. Three unit vectors parallel to these channels are labeled A, B, and $\mathrm{C}$ in the figure, and are described by Equation (3.1) in matrix form. On the existing sensor, all channels point at an angle of $\theta_{o}=-67.5^{\circ}$ down from the $\hat{x}, \hat{y}$ plane, and are separated (clockwise from $\hat{x}$ ) by $\theta_{A}=0^{\circ}, \theta_{B}=240^{\circ}$, and $\theta_{C}=120^{\circ}$. Unit vectors parallel to each channel can be entered into a matrix form as

$$
[S]=\left[\begin{array}{ccc}
\cos \left(\theta_{o}\right) & 0 & \sin \left(\theta_{o}\right) \\
\cos \left(\theta_{o}\right) \cos \left(\theta_{B}\right) & \cos \left(\theta_{o}\right) \sin \left(\theta_{B}\right) & \sin \left(\theta_{o}\right) \\
\cos \left(\theta_{o}\right) \cos \left(\theta_{C}\right) & \cos \left(\theta_{o}\right) \sin \left(\theta_{C}\right) & \sin \left(\theta_{o}\right)
\end{array}\right]\left[\begin{array}{l}
\hat{x} \\
\hat{y} \\
\hat{z}
\end{array}\right]
$$


The matrix described by (3.1) becomes very useful for solving velocity vectors, and platform attitude.

\subsection{Vector Velocity Computation}

Given that the sensor platform is moving at a magnitude $|\vec{V}|$ and a direction $\vec{V}=v_{x} \hat{x}+v_{y} \hat{y}+v_{z} \hat{z}$ then the measured LOS velocities $M_{A}, M_{B}$, and $M_{C}$ for channels $A, B$, and $C$ respectively are obtained from the dot-products of the DL channel unit vectors and the velocity vector

$$
\left[\begin{array}{l}
M_{A} \\
M_{B} \\
M_{C}
\end{array}\right]=[S] \cdot\left[\begin{array}{c}
v_{x} \\
v_{y} \\
v_{z}
\end{array}\right]
$$

Equation (3.2) provides three equations with the three unknown velocity components $v_{x}, v_{y}$, and $v_{z}$, that comprise the velocity vector $\vec{V}$, and can therefore be solved simultaneously to high accuracy. When range information is not required, velocity vectors can be obtained directly from the LOS Doppler measurements without the need of any waveform modulation. For this reason, three of the six flights were conducted without the modulation function turned on.

The velocity components of the solved velocity vector are given in the DL coordinate frame of reference. In order to obtain the velocity components in the landing site frame of reference or any other frame of reference, one must have rotation-transformation knowledge to that reference.

\subsection{Vehicle Altitude, and Attitude Computation}

As discussed in the waveform description section, measurements of range can be made to high precision ${ }^{3}$. Added to the range measurement capability is the availability of three channels, as defined in the geometric description. Range information from the three channels can be used to extract vehicle altitude above ground level (AGL), and vehicle attitude relative to the ground reference frame.

For a vehicle traveling in the positive $\mathrm{x}$-direction, platform attitude refers to roll, which is rotation of the vehicle along the $\mathrm{x}$ - axis, pitch, rotation along the $\mathrm{y}-$ axis and yaw $(\gamma)$, rotation along the $\mathrm{z}-$ axis. Roll and pitch can be computed from the LOS range measurements. Side slip angle (SSA) is the angle between the nose of the vehicle, and the direction of travel. For the assumption that the platform and sensor $\mathrm{x}$-axes are identical, a similar parameter can be given for angle of approach (AoA) which is defined as the angle made by the $\mathrm{x}$-axis of the platform and the direction of travel.

\section{TEST DESCRIPTION}

Flight tests were performed at NASA Dryden on August $20^{\text {th }}$ thru $22^{\text {nd }}$, 2008. There were a total of six flights performed during this campaign. Flights 1, 3, and 5 were made with the waveform modulation off, which means that only Doppler LOS velocities were collected for these flights. In contrast, flights 2, 4, and 6 were made using the FMCW waveform so that in addition to LOS velocities, LOS range measurements were also acquired. Flights 1 through 4 were made over flat terrain, while Flights 5 and 6 were made over hilly and rough terrain.

During takeoff and landing, the gimbal is locked in a stowed position in order to prevent debris lifted by the helicopter propeller from damaging the optical components of the DL. This stowed position is maintained until the helicopter is positioned over the surveyed test area. Once at the test area, the gimbal is moved so that the center axis of the DL points towards nadir as described in the geometrical description section of this paper. The intent was to maintain nadir lock over the duration of the flights. However, due to control software issues, the gimbal had to be returned to a stowed 
position at the ends of each "figure -8 " in the flight trajectories. Gimbal motion at the ends of the trajectory

complicated the data analysis portion of the test. However, data collected during these times proved to be just as valuable as the data collected while the gimbal was in a nadir lock position.

\section{RESULTS}

The following section summarizes the results obtained from the DL system. The two modes of operation of the DL that were tested at NASA Dryden are Doppler only from the CW waveform and simultaneous Doppler/Range measurements from the FMCW waveform.

\subsection{CW Mode}

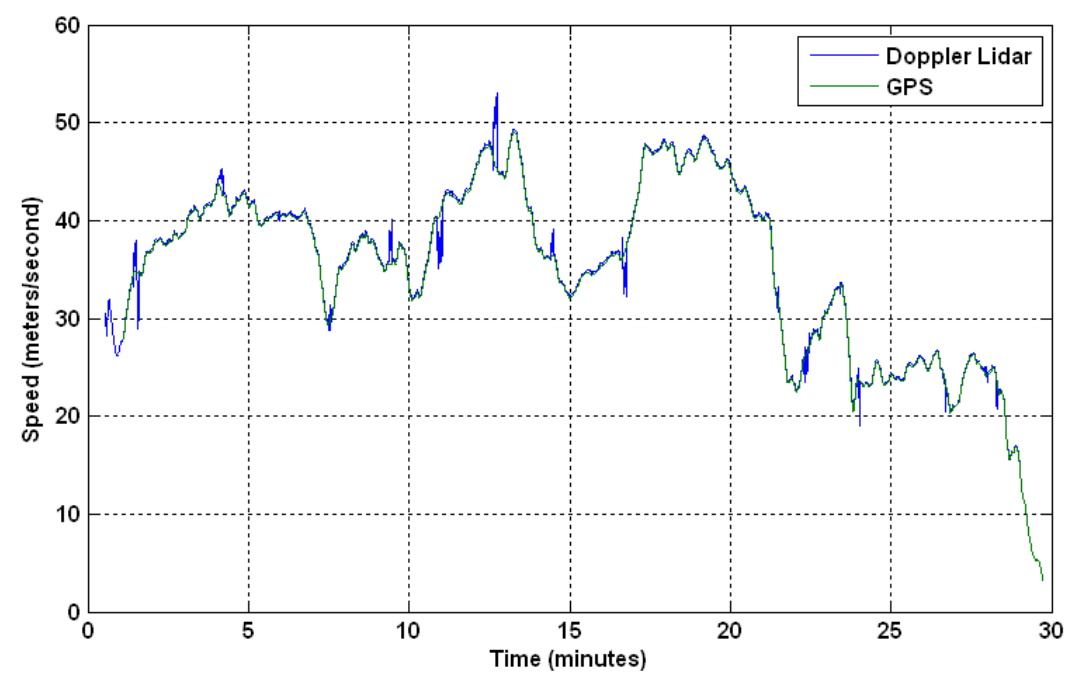

Figure 5 Velocity magnitude comparison between the NDGPS data and the DL measurements.

The CW mode operation provided very high signal to noise ratios for all three flights (Flights 1, 3, and 5), even during the stowed position periods when the lidar was pointed towards the horizon and the range to the ground was substantially

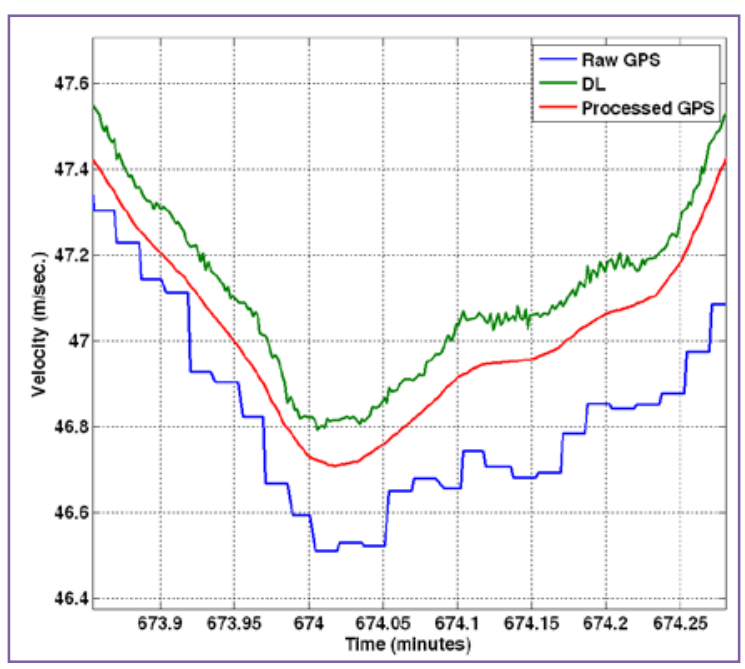

Figure 6 Expanded view shows details of the data presented by Figure 8, and including the raw GPS data. increased. In fact, there are ample signal levels for all altitudes flown during this campaign in both operational modes. The tests show that no measurable difference exists between velocity measurements obtained over a flat terrain and velocity measurements made over rough or uneven terrain. This stands to reason since the measurement process comes from the Doppler effect and not from the time of flight of a signal as is the case of pulsed range-rate lidar or radar measurements.

A good example of the performance of the CW mode of operation is provided by Flight 5. Maximum altitude was limited to the helicopter ceiling, which for Flight 5 was approximately 1,820 meters ASL. Assuming ground elevation of 670 meters, this altitude corresponds to a LOS range of approximately 1,245 meters per channel during nadir lock. At the highest altitudes, the SNRs greater than $10^{5}$ are readily obtained. To get an operational range estimate for this breadboard system on the CW mode of the DL, the available data was used to make an extrapolation of the

SNR curve versus altitude, indicating operational platform altitudes of over 5 kilometers. Figure 5 is a plot comparing 
the velocity magnitudes of the numerically derived from GPS (NDGPS) data to the velocity magnitude measured by the DL. Figure 6 is an expended view of a portion of Figure 5 data showing the raw GPS data, the NDGPS velocities, and the DL velocity measurements. There is a small offset of about $10 \mathrm{~cm} / \mathrm{sec}$ between the DL and the NDGPS measurements that may be attributed to a bias error in GPS data. The numerically derived GPS velocity data are estimated to have less than $3 \mathrm{~cm} / \mathrm{sec}$ noise, but its bias error is not clearly understood at this time.

To compare the velocity vector of the DL to the velocity vector produced by the NDGPS, the velocity components are plotted simultaneously. Results for Flight 5 are shown in Figure 7, showing excellent agreement of the velocity vector

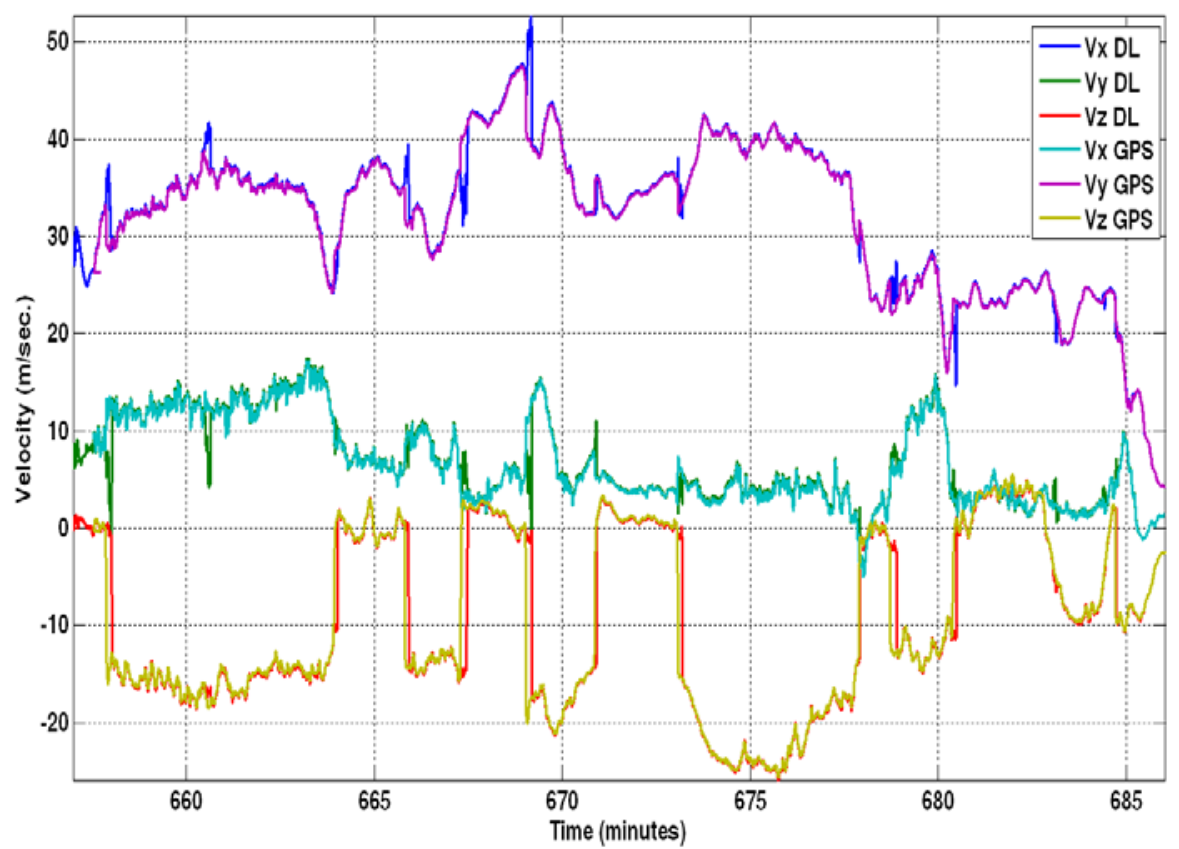

Figure 7 Velocity vector components obtained by the DL are compared to NDGPS velocity component data.

components between the two systems. The DL data shows spikes in velocity several times during each flight. These spikes are caused by the motion of the gimbal when going from locked nadir position to stowed position. These velocity spikes are not detected by the GPS receiver since the GPS only tracks the helicopter motion. A more quantitative comparison can be made by taking the cross correlation of the components. The cross correlations between the velocity components, including the gimbal motion, are $99.28 \%$ for $\mathrm{Vx}, 97.94 \%$ for $\mathrm{Vy}$, and $98.51 \%$ for $\mathrm{Vz}$, over the duration of the flight indicating excellent agreement. 


\subsection{FMCW Mode}
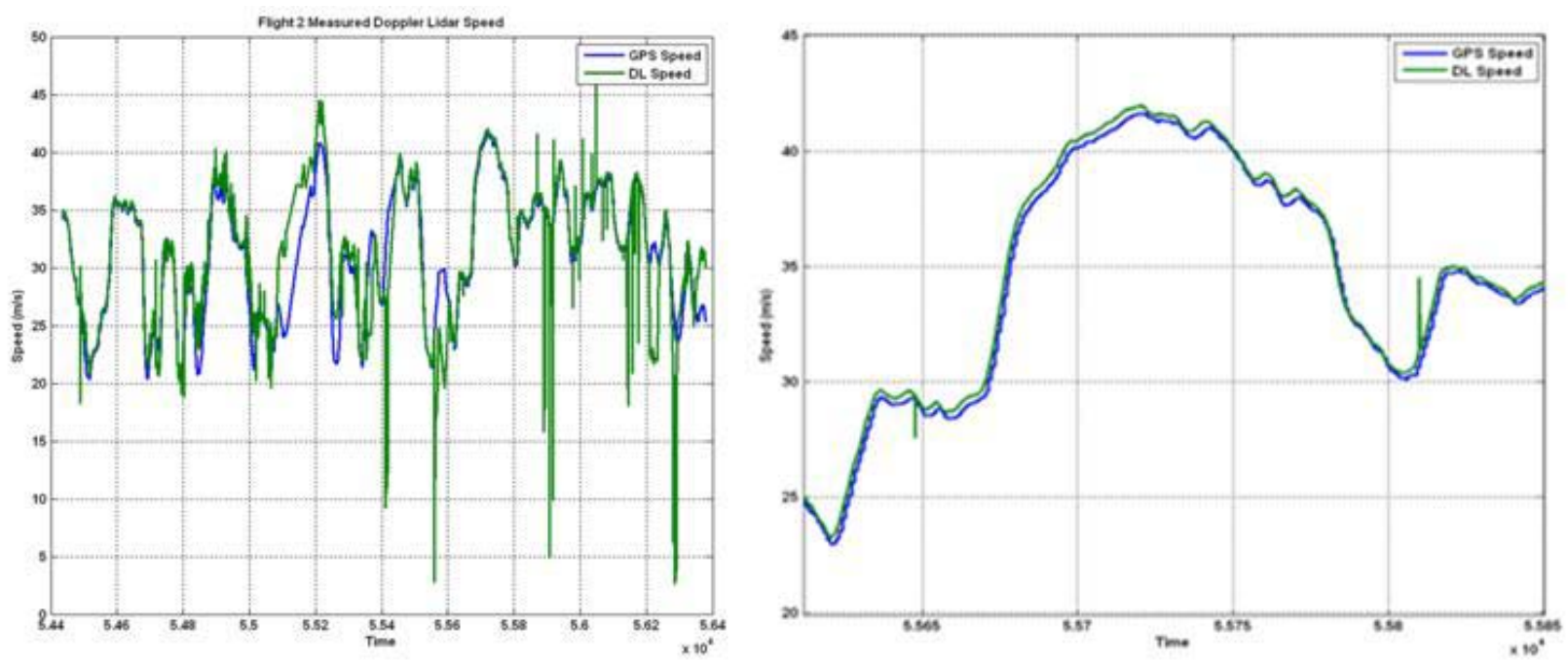

Figure 8 FMCW mode velocity comparison to GPS for Flight 2 (left). Right figure is an expanded view of the velocity magnitude comparison.

The FMCW mode of operation is complex when compared to the CW mode. However, a significantly greater amount of information can be extracted from the signals when this waveform is used. All measurements made by the DL rely on the knowledge of the IF, regardless of the mode of operation (CW, FMCW). For this reason, the majority of the signal processing and analysis is performed in the frequency domain. To distinguish and identify the signals from the noise, an algorithm was developed that removes the background noise present at each of the three receivers, and optimizes the SNR. Once this algorithm is applied to the received signal, the frequencies associated with the waveform's up- ramps and down-ramps are then identified, from which range and velocity is obtained. Figure 8 is a comparison between GPS and the DL for Flight 2, when the DL was operating in the FMCW mode. An expanded view of the velocity is shown on the right plot of Figure 8. The added noise and the perceived reduced accuracy shown in the left plot are due to the signal processing algorithm used, and limitations on some of the hardware components of this breadboard system. The FMCW results, which show excellent correlation between the DL data and GPS, are consistent with the CW mode measurements.

\section{ALTITUDE AND GROUND RELATIVE ATTITUDE MEASUREMENTS}

In addition to velocity measurements, the FMCW mode allows the DL to obtain LOS range measurements from each of the three channels. The ability to measure LOS range on each channel of the DL provides sufficient information to compute altitude above ground level (AGL), and vehicle attitude relative to the ground.

Figure 9 is a plot of the helicopter altitude obtained by the DL from the LOS range measurements compared with GPS altitude (blue curve). Since GPS provides altitude measurements relative to sea level, a ground elevation value was used to obtain a best fit of the two data sets. As noted in the velocity measurement results, the noise is due to the signal processing algorithm and not to the DL capabilities. Noise is more pronounced at the lower altitudes because of high SNR causing receiver saturation. Figure 10 shows details of the altitude measurements for this flight. The plot on the left covers approximately 12 minutes of the flight. Four different gimbal movements from nadir to the stowed position occurred during this time, showing that it is not important to point nadir in order to measure platform altitude directly above ground level. The right plot corresponds to 36 seconds that cover the ceiling of the flight at $1.03 \mathrm{~km}$ altitude above ground level. Recall that the DL altitude is measured relative to the local terrain by defining a reference plane containing the three laser spots on the ground. On the other hand, the GPS altitude is measured relative to global sea level and corrected by applying a nominal ground level. Therefore the GPS data does not recognize the local terrain features. This 


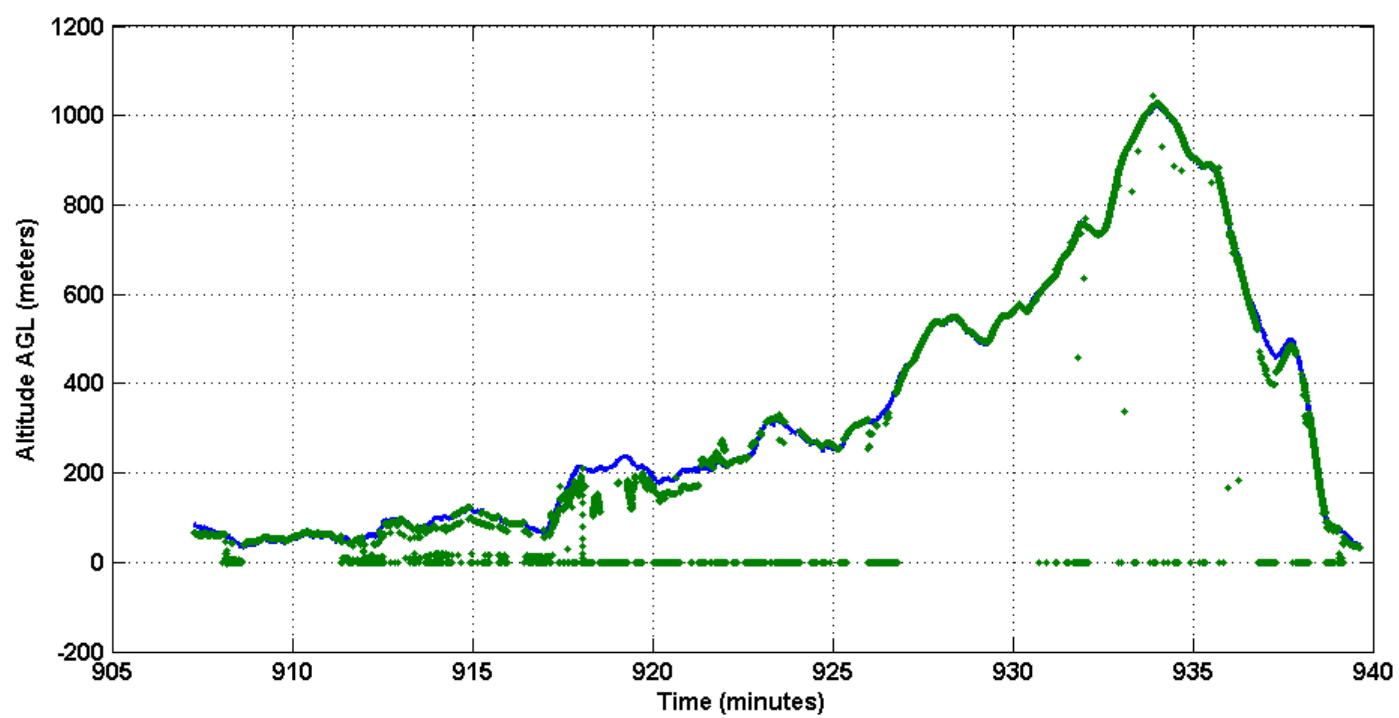

Figure 9 Flight 2 Altitude measurements. The blue trace is the GPS altitude data; the green points mark the measured altitude by the DL.
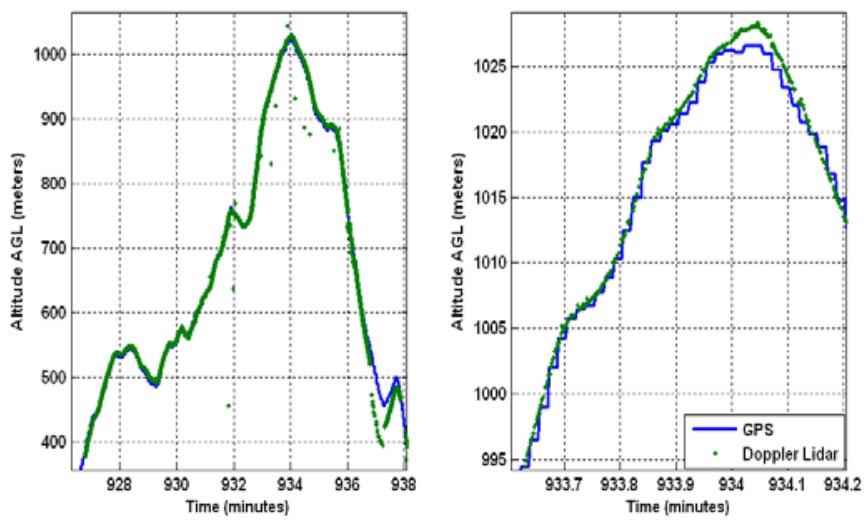

Figure 10 Expanded views of the altitude measurements for Flight 2. Left figure is 12 minute window and right figure is 36 second window crossing the highest altitude of $1.03 \mathrm{~km}$ above ground level.

fact makes it difficult to compare the two altitude measurements, however in spite of the GPS limitations, the data agrees very well to within a meter at $1.03 \mathrm{~km}$ altitude.

Altitude above ground level is obtained from the complete knowledge of the plane defined by the LOS range measurements. That same knowledge can now be applied to measure the vehicle's attitude relative to the ground plane. The left plot of Figure 11 shows is a plot of Roll and Pitch angles measured at the times corresponding to the detail plot of Figure 10 (left). During nadir lock position, roll and pitch are zero due to gimbal control and flat terrain. When the gimbal is moved to the stowed position, the gimbal is inactive and the sensor is stationary relative to the helicopter. At this point, the roll and pitch angles correspond to the helicopter's attitude relative to the ground plane. The roll angle changes as the helicopter turns in preparation of the next pass over the surveyed target area, while the pitch angle is fairly constant. 

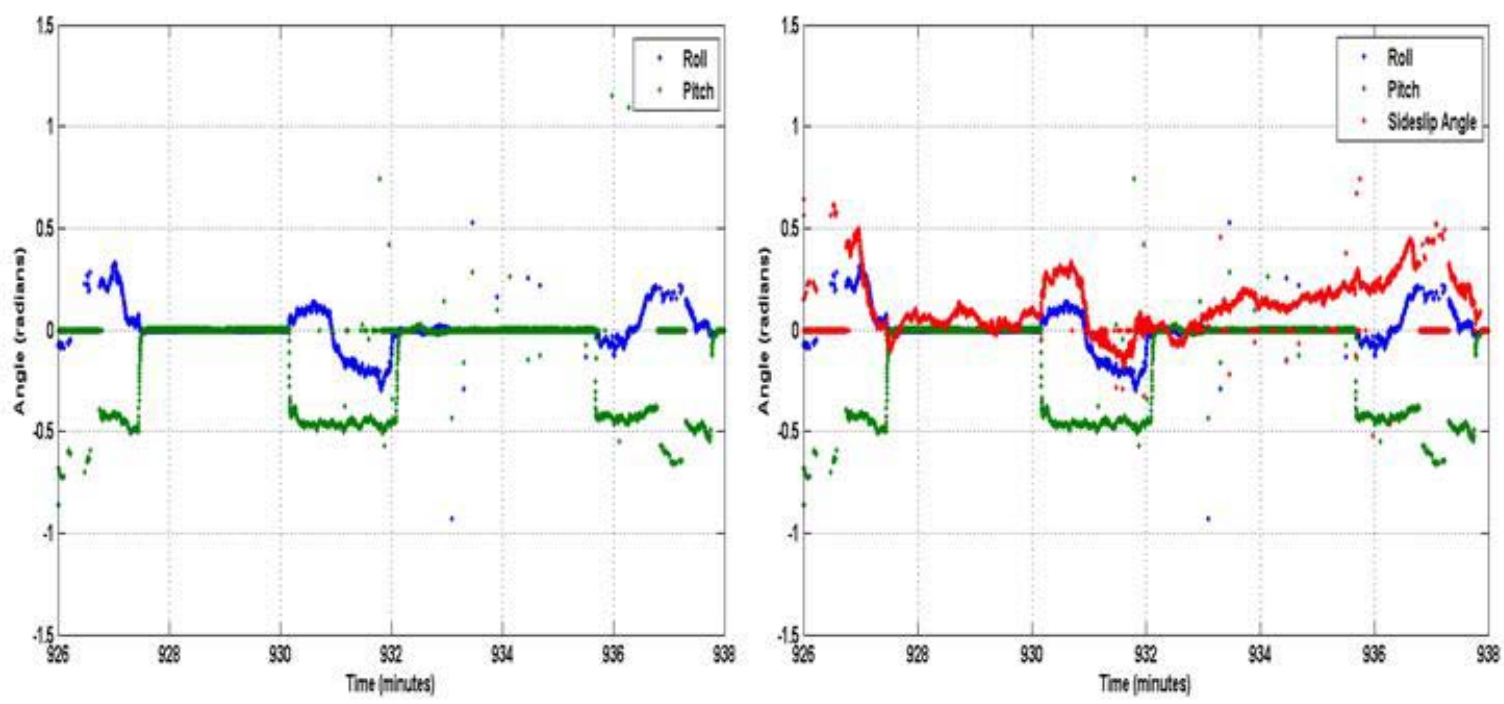

Figure 11 Roll and Pitch for a portion of Flight 2 (left). Zero roll and pitch occurs when in nadir lock position. Roll, pitch and SSA plotted together for the same period of flight (right).

Once roll and pitch is applied to the DL measurements, the sideslip angle can be computed from the velocity vectors. Sideslip angle is the angle between the nose of the helicopter and the direction of travel. The right plot of Figure 11 shows roll, pitch, and sideslip angle for the same time window as the left plot.

\section{CONCLUSIONS}

An all-fiber navigation Doppler Lidar was tested aboard a helicopter over different terrains, altitudes, and velocities. The post processing of the lidar data indicates excellent agreement to the numerically derived GPS data. This helicopter flight campaign proved that this Doppler lidar can be a very valuable sensor for future NASA landing missions by providing critical vehicle velocity, altitude, and attitude data with a high degree of accuracy. The data obtained and the lessons learned from this field test are also a valuable asset for the development of the next generation prototype unit that is currently under development at NASA Langley Research Center, in support of the ALHAT project.

\section{ACKOWLEDGMENTS}

The authors would like to acknowledge the contributions of NASA Jet Propulsion Laboratory ALHAT team in particular Sohrab Mobasser, Jason Keim, Ed Konefat, Dave Natzic for the helicopter flight tests support and data processing of the flight trajectories, and a special thank you to the sensor operator Garen Khanoyan. The authors are also thankful to the ALHAT project manager, Chirold Epp, NASA Johnson Space Center for his support.

\footnotetext{
${ }^{1}$ Pierrottet, D. F., F. Amzajerdian, BL Meadows, R. Estes, A. Noe, “Development of an All-Fiber Coherent Laser Radar for Precision Range and Velocity Measurements", Advanced Devices and Materials for Laser Remote Sensing, Materials Research Society, Materials and Devices for Laser Remote Sensing and Optical Communications. San Francisco (2005).

2 Amzajerdian, F., D. Pierrottet, "Development of a Coherent Lidar for Aiding Precision Soft Landing on Planetary Bodies,” Proceedings of 13th Coherent Laser Radar Conference, Kamakura, Japan, (2006).

${ }^{3}$ Pierrottet, D. F., F. Amzajerdian, L. Petway, B. Barnes, G. Lockard, "Linear FMCW Laser Radar for Precision Range and Vector Velocity Measurements", Material Research Society. Materials and Devices for Laser Remote Sensing and Optical Communications, San Francisco, (2008).
} 